\title{
Erratum to: The WHO ultrasonography protocol for assessing morbidity due to Schistosoma haematobium. Acceptance and evolution over 14 years. Systematic review
}

\author{
Robert Akpata ${ }^{1,2}$ • Andreas Neumayr ${ }^{4}$ - Martha C. Holtfreter ${ }^{2}$. \\ Ingela Krantz ${ }^{3}$ Daman D. Singh ${ }^{2} \cdot$ Rodrigo Mota $^{2}$ • \\ Susanne Walter ${ }^{6}$ Christoph Hatz ${ }^{4,5}$. Joachim Richter ${ }^{2}$
}

Published online: 27 March 2015

(C) Springer-Verlag Berlin Heidelberg 2015

Erratum to: Parasitology Research

DOI: $10.1007 / \mathrm{s00436-015-4389-z}$

The original article "The WHO-ultrasonography protocol for assessing morbidity due to Schistosoma haematobium. Acceptance and evolution over 14 years. Systematic review"

The online version of the original article can be found at http://dx.doi.org/ 10.1007/s00436-015-4389-z.

\footnotetext{
Joachim Richter

Joachim.Richter@med.uni-duesseldorf.de

1 Hôpital Saint Jean de Dieu, Tanguieta, Benin

2 Tropical Medicine Unit, Department for Gastroenterology, Hepatology and Infectious Diseases, Medical Faculty, HeinrichHeine-University Düsseldorf, Düsseldorf, Germany

3 Skaraborg Institute for Research and Development, Skövde, Sweden

4 Swiss Tropical and Public Health Institute, Basel, Switzerland

5 Institute for Social and Preventive Medicine, University of Zürich, Zürich, Switzerland

6 Institute of Biology and Zoomorphology, Faculty of Biology, Heinrich-Heine-University Düsseldorf, Düsseldorf, Germany
} 
contained some mistake in Figure 1 which we would like to correct. The revised Figure 1 is indicated below:

PubMed search 'ultrasound' OR ultrasonography (US)' AND 'urinary schistosomiasis' [2001-2014]

71 identified publications

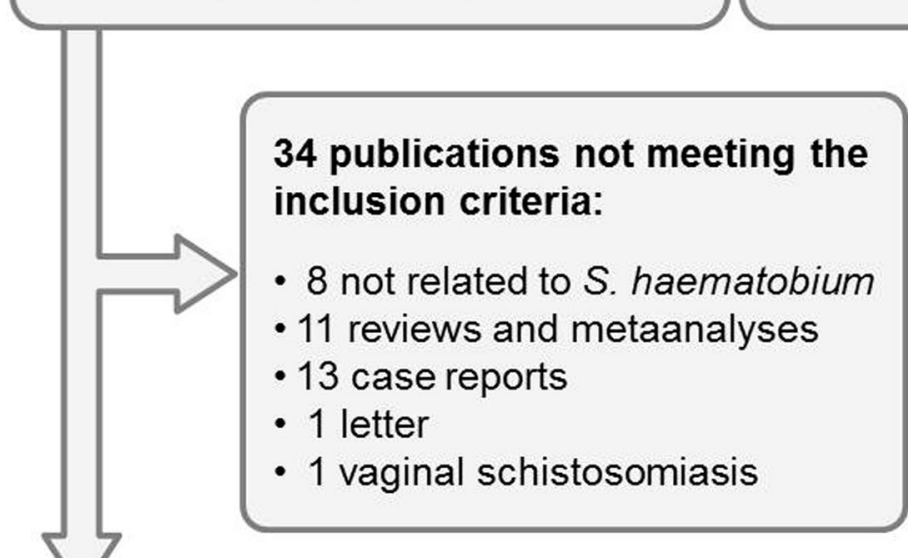

34 publications not meeting the criteria:

8 not related to $S$. haematobium

- 11 reviews and metaanalyses

- 13 case reports

- 1 letter

- 1 vaginal schistosomiasis
PubMed search 'ultrasound' OR ultrasonography (US)' AND 'S. haematobium' [2001-2014]

\section{9 identified publications}

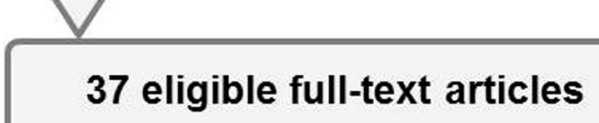

33 eligible full-text articles

30 duplicates / identical articles excluded

40 full-text articles (on 17861 patients) assessed for data

1 article reporting results of 2 different studies

3 articles excluded referring to same data

37 full-text articles reporting on 38 studies (on 17861 patients) included in the analysis 